\title{
Experimental study on wave dissipation coefficient for tetrahedron
}

\section{penetrating frames breakwater}

\author{
Shu Zhang ${ }^{1, a}$, Zi-chang Shang-guan ${ }^{2, b}$, li-hua Huang ${ }^{3, c}$, and Jia-ying Jing ${ }^{4, d}$ \\ 1 Dalian Ocean University,Dalian 116023,China \\ 2 Dalian Ocean University,Dalian 116023,China \\ 3 Dalian University of Technology,Dalian116023,China \\ 4 Dalian Ocean University,Dalian 116023, China \\ a1010517204@qq.com, ${ }^{\text {b }}$ shangguan@dlou.edu.cn, ${ }^{\mathrm{c}} 409450427 @ q q . c o m$, d371075187@qq.com
}

Keywords: tetrahedron penetrating frames; experimental study; wave attenuation performance; transmission coefficient

Abstract.Tetrahedron penetrating frames is a new breakwater structure.In this paper,the attenuation characteristics of tetrahedron penetrating frames were measured through physical modeling. The effect of the wave period,wave height,the number of rows, underwater penetration, row space,layout,and other factors on the wave transmission coefficient were investigated. The results show that the proposed breakwater has good wave dissipation capacity, which has broad application prospects.

\section{Introduction}

Coastal zone has special geographical position and abundant natural resources, which plays an important role in social development. Along with the global climate change and human activities, the coastal erosion problems are becoming increasingly serious. Global $440000 \mathrm{~km}$ coastline that $2 / 3$ above is affected by different degrees of erosion, sandy coast was erosion more than 70\%[1].This situation has seriously affected the social and economic development in the coastal areas. Therefore, it is an important issue to study the protective measures of coastal zone. There are some inherent defects in the traditional breakwater, such as high cost, complex technology, construction difficulties, etc[2]. Tetrahedron penetrating frame is a kind of new structure of economical and environmental protection, which is a component that has the effect of reducing, guiding, eliminating energy, promoting the deposition, etc[3,4].The structure has good balance, simple structure and low cost. In addition, it has strong adaptability to the terrain, and does not need the foundation treatment. When the seabed condition is poor, the tetrahedron penetrating frame is a relatively good structure[5,6].

The structure of tetrahedron penetrating frame is put forward by Gao Wen-he[7], which was attended more attention in recent years. At present, some achievements have been made in the research on tetrahedron penetrating frame[8,9], which was mainly confined to the tetrahedron penetrating frame of river bank protection applications.Wu Long-hua[10] proposed that the structure can not only be used in the protection of the Bank of the rivers and lakes, but also has a good application prospect in the protection of the beaches.In the light of the current domestic use of tetrahedron penetrating frame work for coastal protection research results are less, and there is not enough theoretical guidance for engineering application, the paper carried out the study of physical 
model experiment. Analyzed the cycle, wave height, arrangement and other factors on the tetrahedron penetrating frame structure of the influence of wave transmission coefficient, and the model is optimized, which provides reference for engineering application.

\section{Physical Model}

Experiment Facilities and Instruments. The experiment is carried out in a wave flume in the Key Laboratory (length $40 \mathrm{~m}$, width $0.7 \mathrm{~m}$, height $1 \mathrm{~m}$ ) of coastal engineering in Liaoning, Dalian. At one end of the flume, a wave generating machine is provided with a one way wave and irregular wave, and the waveform is stable and reproducible; at the other end of the flume is provided with the wave-dissipation device, which is used to absorb the wave energy to reduce the wave reflection. The automatic acquisition and analysis of the experimental data is controlled by the microcomputer system, the wave measurement using DS30 type 64 channel wave height gauge.

Experiment Conditions. Test used regular incident waves, with the depth of $d=0.3 \mathrm{~m}$, height of $\mathrm{H}=0.07 \mathrm{~m}, \mathrm{H}=0.09 \mathrm{~m}$ two groups of wave height, wave periods were 0.8, 0.85, 0.9, 1, 1.05, 1.1, 0.95, $1.15,1.2 \mathrm{~s}$; model text on the scale of $1: 30$, which respectively corresponding to the actual height is $\mathrm{H}=2.1 \mathrm{~m}, \mathrm{H}=2.7 \mathrm{~m}$, the corresponding actual periods were $4.38,4.66,4.93,5.48,5.75,6.02,5.20$, $6.30,6.57 \mathrm{~s}$.

Experiment model.The use of the tetrahedron penetrating frame in engineering is a prefabricated reinforced concrete member, which is connected by six root length equal rods. According to the experimental facilities and prototype conditions, the model scale is 1:30. The coastal protection wave velocity and wave energy is far greater than the river revetment, so the width of the bar in the six sided water frame model is optimized.Three pyramid model experiment used a square cross-section hollow pipe welded,the tube filled cement, to increase the weight.The section size of the bar is $25 \mathrm{~mm} * 25 \mathrm{~mm}$, the wall thickness is $1 \mathrm{~mm}$, and the length of each member is $180 \mathrm{~mm}$, as shown in Fig. 1.The flume model meets the similar geometry and flow pattern.

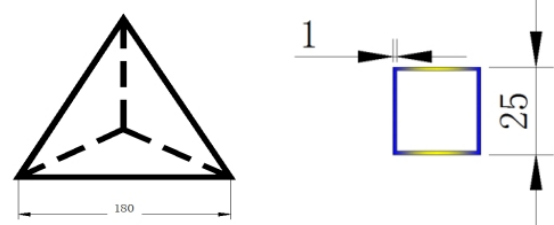

Fig.1 Tetrahedron-like penetrating frame model

Experimental Setup.As shown in Fig. 2,that at one end of the flume is a wave machine and the other end is a wave-dissipation device.In the experiment, the model is arranged in the middle of the flume. One wave gauge was set up in front of the model,and one in the rear.The wave height gauge which layout in the front of model for $2 \mathrm{~m}$ distance used to measure the hight of incident waves at breakwater anterior and the wave height gauge which layout behind the model for $1.8 \mathrm{~m}$ distance used to measure the height of incident waves at breakwater anterior.The sampling interval was $0.02 \mathrm{~s}$, and 1024 data increments were collected.The wave data from the two gauges were analyzed by the reflection coefficient $(\mathrm{Kt}=\mathrm{Ht} / \mathrm{Hi})$ was gained.The model layout scheme is shown in Table 1.

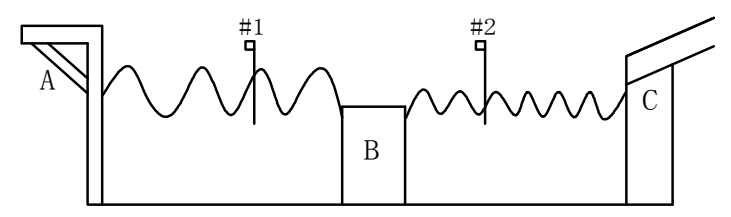

A:Wave maker B:Model C:Wave-dissipation device \#1 \#2:Wave height gauges

Fig.2 Experiment Setup 
Table 1 Model layout scheme

\begin{tabular}{cccccc}
\hline Layout scheme & Water depth $/ \mathrm{d} \cdot \mathrm{m}^{-1}$ & $\begin{array}{c}\text { The number of } \\
\text { layer/n }\end{array}$ & $\begin{array}{c}\text { Water } \\
\text { depth } / \mathrm{d}_{1} \cdot \mathrm{m}^{-1}\end{array}$ & $\begin{array}{c}\text { The number } \\
\text { of row/n }\end{array}$ & $\begin{array}{c}\text { Separation } \\
\text { distance } / \mathrm{d}_{2} \cdot \mathrm{m}^{-1}\end{array}$ \\
\hline Plan one & 0.3 & 2 & 0 & $4 \sim 10$ & 0.4 \\
Plan two & $0.35 \sim 0.25$ & 2 & $-0.05 \sim 0.05$ & 8 & 0.4 \\
Plan three & 0.3 & 2 & 0 & 8 & $0 \sim 0.6$ \\
\hline
\end{tabular}

\section{Analysis of Test Result}

The article focuses on wave attenuation performance by the tetrahedron penetrating frames. The wave attenuation performance including wave cycle, wave height,row number of frames, depth of enter the water, row spacing and the influence of different layouts for transmission coefficient $\mathrm{K}_{\mathrm{t}}$. The espression of transmission coefficient $\mathrm{K}_{\mathrm{t}}$ :

$\mathrm{K}_{\mathrm{t}}=\mathrm{H}_{\mathrm{t}} / \mathrm{H}_{\mathrm{i}}$

among that the $\mathrm{H}_{t}$ means hight of transmitted waves penetrat breakwater, the $\mathrm{H}_{\mathrm{i}}$ means hight of incident waves at breakwater anterior.

Effect of wave cycle $\mathbf{T}$ on transmission coefficient.Fig.4 to Fig.9 show that test results of transmission coefficient by tetrahedron penetrating frames under different wave cycles.It can be seen from the figures that the influence of wave cycle on the transmission coefficient is obvious. The wave transmission coefficient increases with the increase of the wave cycle,so that the effect of wave attenuation is poor when the incident waves with short cycles.

Effect of layout number on transmission coefficient.Physical model tests are carried out for observation of the wave dissipation performance of layout plan one. Fig. 4 comparison of the experimental results of the transmission coefficient under different layout which was divided into four cases just like 4,6,8 and 10 rows.Each additional arrangement to increase the length of $0.58 \mathrm{~m}$. The test results show that the transmission coefficient decreases with the increase of the number of rows, and the change trend is obvious. With the increase of the number of rows, the effect of wave dissipation is significantly improved, especially for the short period. From Figure 3, when the layout of the 4 rows (the length is $1.92 \mathrm{~m}$ ) and the period was more than $1 \mathrm{~s}$, about $90 \%$ of the wave through. That indicate if the the length of layout is too short the effect of wave dissipation is poor.

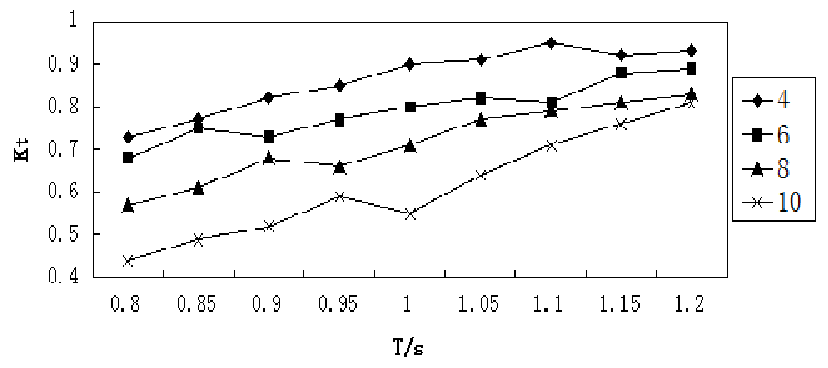

(a) $\mathrm{H}=0.07 \mathrm{~m}$

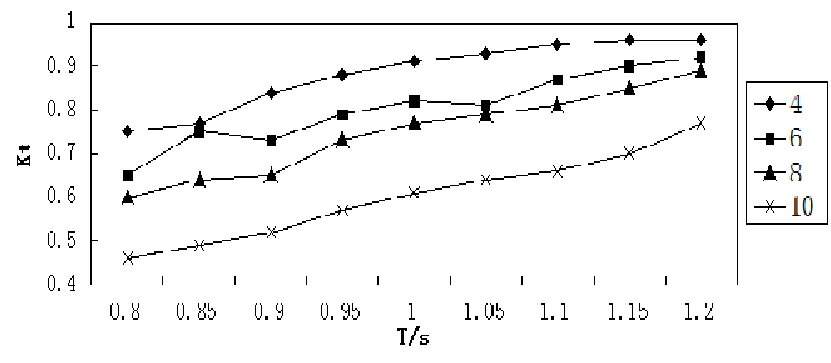

(b) $\mathrm{H}=0.09 \mathrm{~m}$

Fig.3Effect of layout number on transmission coefficient

Effect of water depth on transmission coefficient.Physical model tests are carried out for observation of the wave dissipation performance of layout plan two. A note about this case is that the height of the 2 layer model is certain $(0.3 \mathrm{~m})$, when the water depth was $0.35 \mathrm{~m}, 0.3 \mathrm{~m}$ and $0.25 \mathrm{~m}$ that the model is corresponding to the water $0.05 \mathrm{~m}$, the model has just been flooded and the model is exposed to the surface $0.05 \mathrm{~m}$. Fig.4shows the influence of different water depth on the 
transmission coefficient, the influence of water depth on the transmission coefficient is obvious, and the transmission coefficient increases with the increase of the water depth. It can be seen that the water depth $0 \mathrm{~m}$ and $-0.05 \mathrm{~m}$ of the wave dissipation effect is almost no difference, the water depth of the $0 \mathrm{~m}$ (model has just been flooded) can achieve the best results.

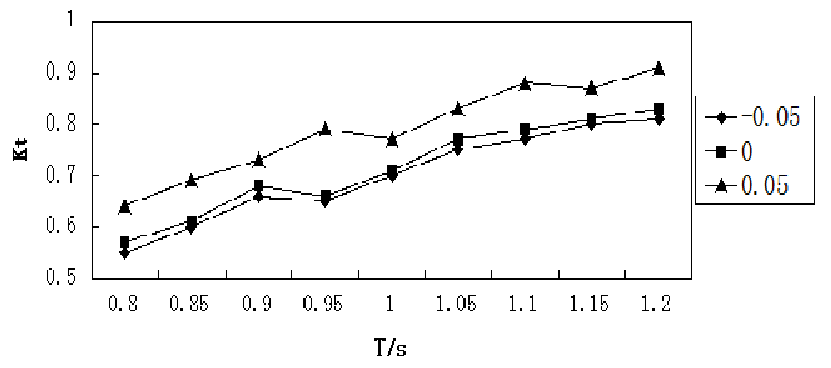

(a) $\mathrm{H}=0.07 \mathrm{~m}$

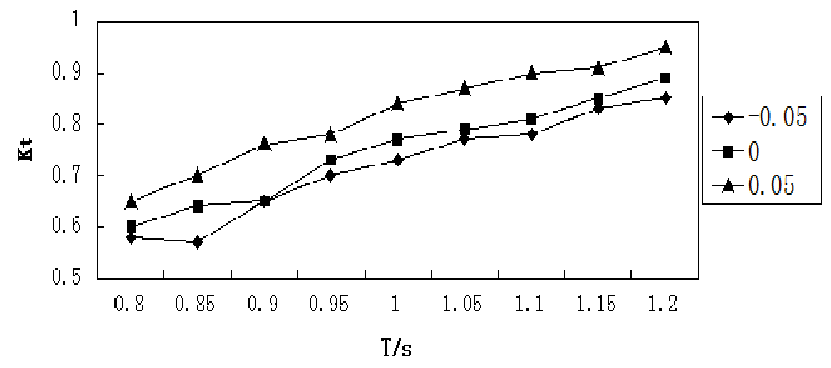

(b) $\mathrm{H}=0.09 \mathrm{~m}$

Fig.4Effect of water depth on transmission coefficient

Effect of row spacing on transmission coefficient.Physical model tests are carried out for observation of the wave dissipation performance of layout plan three. Fig. 6 shows the experimental results of the influence of the distance between the row spacing on the transmission coefficient, and the row spacing is divided into four cases: $0.2 \mathrm{~m}, 0.4 \mathrm{~m}, 0 \mathrm{~m}$ and $0.6 \mathrm{~m}$. From the Fig. 5 when the distance is $0 \mathrm{~m}$ the transmission coefficient is very large so the effect of wave dissipation is poor, and the transmission coefficient decreases with the increase of the row spacing. When the distance change from $0.4 \mathrm{~m}$ to $0.6 \mathrm{~m}$ the transmission coefficient increases and the dissipation effect becomes worse, which shows that the $0.4 \mathrm{~m}$ is the best.

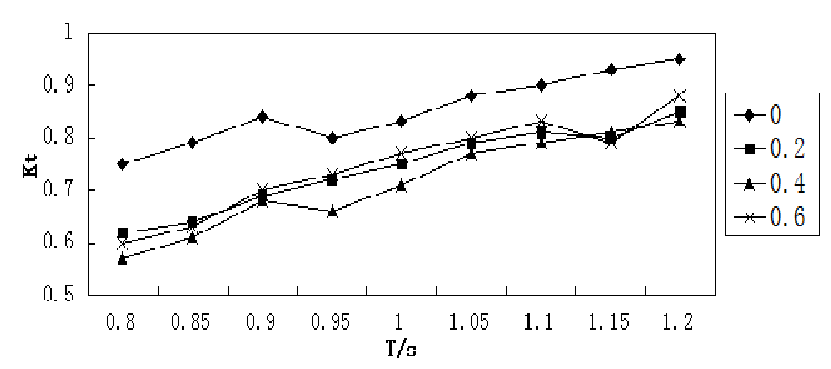

(a) $\mathrm{H}=0.07 \mathrm{~m}$

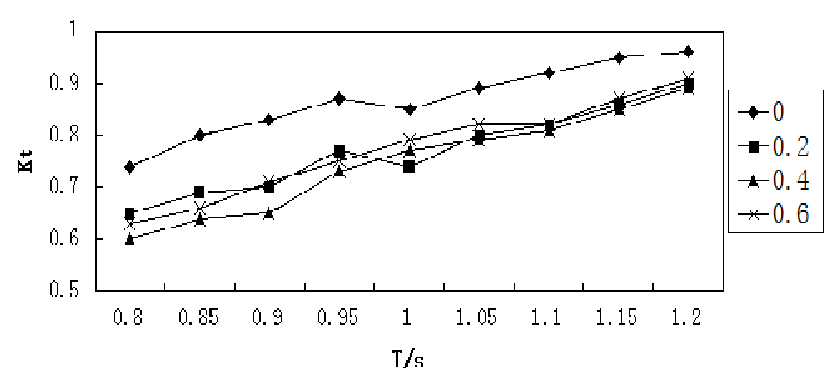

(b) $\mathrm{H}=0.09 \mathrm{~m}$

Fig.5Effect of row spacing on transmission coefficient

Effect of rule set and random set on transmission coefficient. Fig.6shows that the experiment results of the influence of the transmission coefficient on the two cases of rule set and random set.It can be seen from Fig.9 that the transmission coefficient of the rule set is reduced by about 5\% 13\% than random set.It shows that the wave dissipation effect of rule set is about $5 \%$ 13\% better than random set.Also from the chart can be seen, with the increase of wave height and wave period, the wave dissipation effect of rule set improve more obvious. 


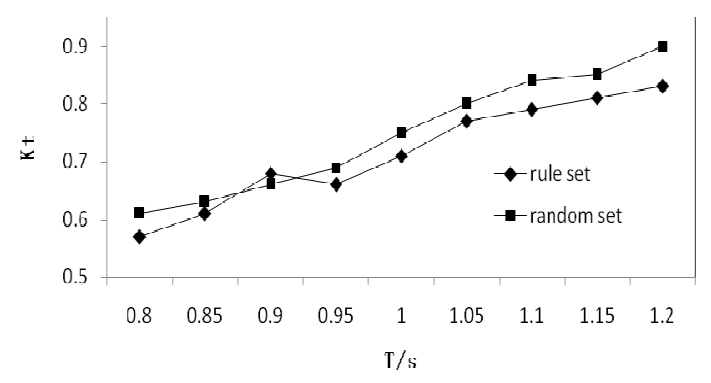

(a) $\mathrm{H}=0.07 \mathrm{~m}$

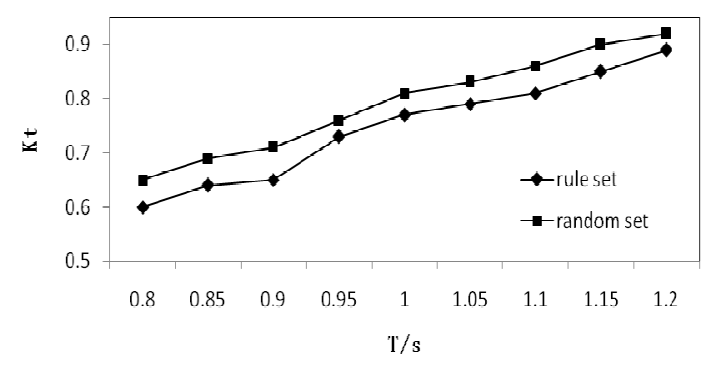

(b) $\mathrm{H}=0.09 \mathrm{~m}$

Fig.6ffect of rule set and random set on transmission coefficient

\section{Conclusions}

In this paper, the transmission coefficient of the incident wave is obtained by using the physical model test to study the dissipation performance of the tetrahedron penetrating frames. The experimental results show that the tetrahedron penetrating frames have a very good effect of wave dissipation.Through adjust the layout of the tetrahedron penetrating frames it can be very good to enhance the effect of wave dissipation.

\section{References}

[1] CHEN Shen-liang,WU Sang-yun,and YU Hong-jun,2006.Discussion on the coastal erosion and protection technology in China,Coastal Engineering,25(1):69-75.(in Chinese)

[2]TANG Hong-wu,LI Fu-tian,and XIAO Yang,2002.Experimental study on effect of scour prevention and sedimentation promotion of bank protection of tetrahedron penetrating frame groups,Port\&Waterway Engineering,344(9):25-28.(in Chinese)

[3]ZHU Jing,FANG Tian,and ZHU Yin,2013.The derivation of retardation efficiency of 4-side hexagon revetment framework group based on energy conservation,Journal of Anhui Agri.Sci,41(28):11577-11579,11583.(in Chinese)

[4]WANG Yong-xue,DONG Hua-yang,and LIU Chong,2010.Experimental study of a pile-restrained floating breakwater constructed of pontoon and plates,China Ocean Engineering,24(1):183-190

[5]YAN Yi-xin,ZHENG Jin-hai, and ZENG Xiao-chuan,1998.Characteristics of wave dissipation for pile-foundation tier-retainer breakwaters, The Ocean Engineering, 16(1):68-74.(in Chinese)

[6]YANG Zhong-hua,BAI Feng-peng,and HU Zhao-yang,2014.PIV experiments of flow around the tetrahedron frame under two different arrangements,Journal Of Basic Science And Engineering,22(5):877-886.(in Chinese)

[7]Gao Wen-he,1998.Use of reinforced concrete frame string closure,Technical Service,26-27(in Chinese)

[8]Martino R,Paterson A,and Piva M,2012.Double-average mean flow and local turbulence intensity profiles from PIV measurements for an open channel flow with rigid vegetation,Environmental Fluid Mechanics,12(1):45-62 
[9]HUAI W X,HAN J,and ZENG Y H,2009. Velocity distribution of flow with submerged flexible vegetation based on mixing-length approach,Applied Mathematics And Mechanics,30(3):343-351.(in Chinese)

[10]Wu Long-hua,2006. Study on Hydraulic characteristics of wake stream of permeable tetrahedron(s) and application,Nanjing:Hohai University.(in Chinese) 